\title{
Análisis de los espectros de absorción de las películas radiocrómicas EBT2 y EBT3
}

\section{Analysis of the absorption spectra of EBT2 and EBT3 radiochromic films}

\author{
León-Marroquín E.Y. ${ }^{12}$, Camacho-López M.A.', García-Garduño O.A. ${ }^{2}$, Herrera-González J.A. ${ }^{2}$, Villarreal-Barajas J.E. ${ }^{3}$, \\ Martínez-Quiroz C.A.', De la Cruz-Pérez D.1, Jiménez-Avilés G.? \\ 'Laboratorio de Fotomedicina Biofotónica Láser de Pulsos Ultracortos, Facultad de Medicina, \\ Universidad Autónoma del Estado de México, Edo. México, México. \\ ¿Laboratorio de Física Médica y Unidad de Radiocirugía, Instituto Nacional de Neurología y Neurocirugía, Cd. México, México. \\ ${ }^{3}$ Department of Oncology and Department of Physics and Astronomy, University of Calgary, Calgary AB, and \\ Department of Medical Physics, Tom Baker Cancer Center, Alberta, Canada.
}

\section{RESUMEN}

Objetivo: Analizar los espectros de absorción neta de las películas radiocrómicas EBT2 y EBT3 para describir su influencia en el comportamiento de las curvas de dosis-respuesta.

Metodología: Las películas se irradiaron en un acelerador lineal de 6 MV. La obtención de los espectros de absorción neta se realizó con espectrofotómetro UV/VIS. Las curvas de dosis-respuesta se obtuvieron con un escáner, un láser He-Ne y un espectrofotómetro.

Resultados: El espectro de absorción de las EBT2 muestra tres bandas de absorción centradas que conservan la posición y aumentan su intensidad en función de la dosis, sin embargo, este comportamiento no se observa en las películas EBT3. La curva dosis-respuesta muestra la máxima sensibilidad utilizando el espectrofotómetro, pero no muestra un comportamiento definido.

Implicaciones: Generación de nuevos conocimientos para la creación de nuevos sistemas ópticos capaces de amplificar la sensibilidad de la respuesta de las películas.

Originalidad: Mostrar la correlación entre los espectros de absorción neta y su influencia en las curvas dosis-respuesta en tres diferentes sistemas ópticos.

Conclusiones: El comportamiento de los espectros de absorción aunado al comportamiento de las curvas dosis-respuesta nos ayuda a descartar el uso de sistemas ópticos que no garanticen un uso clínico confiable.

PALABRAS CLAVE: Espectro de absorción, espectrofotómetro, película radiocrómica. 


\section{ABSTRACT}

Objective: To analyze the net absorption spectra of EBT2 and EBT3 radiochromic films to describe their influence on the behavior of dose-response curves.

Methodology: The films were irradiated in a linear accelerator of $6 \mathrm{MV}$. The net absorption spectra were obtained with a UV / VIS spectrophotometer. Dose-response curves were obtained with a scanner, a He-Ne laser and a spectrophotometer.

Results: The absorption spectrum of the EBT2 shows three focused absorption bands that retain position and increase their intensity as a function of dose, however, this behavior is not observed in EBT3 films. The dose-response curve shows maximum sensitivity using the spectrophotometer, but does not show a defined behavior.

Implications: Generation of new knowledge for the creation of new optical systems capable of amplifying the responsiveness of the films.

Originality: Show the correlation between net absorption spectra and their influence on dose-response curves in three different optical systems.

Conclusions: The behavior of absorption spectra combined with the behavior of the dose-response curves helps to discard the use of optical systems that do not guarantee a reliable clinical use.

KEYWORDS: Absorption spectrum, spectrophotometer, radiochromic film.

\section{Correspondencia}

DESTINATARIO: García-Garduño O.A.

INSTITUCIÓN: Laboratorio de Física Médica y Unidad

de Radiocirugía, Instituto Nacional de Neurología y

Neurocirugía

DIRECCIÓN: Insurgentes Sur \#3877, Col. La Fama, Del.

Tlalpan, C.P. 14269, Ciudad de México, CDMX, México

CORREO ELECTRÓNICO: amanda.garcia.g@gmail.com

\section{Fecha de recepción:}

3 de octubre de 2016

Fecha de aceptación:

15 de diciembre de 2016 


\section{INTRODUCCIÓN}

La película radiocrómica (PR) ha sido diseñada para cubrir las necesidades dosimétricas, tanto en la verificación de dosis para los tratamientos en radioterapia (RT), como en los procedimientos de control de calidad (QA por sus siglas en inglés) ${ }^{[1-3]}$. La PR EBT3 es el último modelo de PR lanzado al mercado por Ashland Specialty Ingredients (Ashland Inc., NJ, USA), la cual es la versión mejorada de la PR EBT2, en donde la capa activa conserva la misma composición de la PR EBT2. Entre las novedades que presenta la película radiocrómica EBT3 están su diseño simétrico para eliminar la dependencia con la cara de escaneo (formada por una sola capa activa, con un espesor de $28 \mu \mathrm{m}$, que se encuentra entre dos capas idénticas de poliéster de $120 \mu \mathrm{m}$ de espesor cada una) y un poliéster mate para evitar la formación de anillos de Newton con el fin de tener mayor precisión en la respuesta. Finalmente, en la capa activa se incorporó un colorante amarillo para disminuir la sensibilidad a la luz ultravioleta (UV) con el objetivo de poder ser utilizadas en QA en cuartos de radioterapia con luz.

La respuesta de la PR a la radiación ionizante se manifiesta por un cambio de color visible. Este cambio de color se caracteriza por la densidad óptica neta $\left(D O_{\text {neta }}\right)$ o la absorbancia neta $\left(A_{\text {neta }}\right)$, la cual se obtiene de la resta entre la respuesta de la película radiocrómica irradiada a cierta dosis y la respuesta intrínseca de la película radiocrómica (la respuesta de la película radiocrómica no irradiada). Por lo tanto, las películas radiocrómicas requieren de un sistema óptico (SO) para medir la respuesta (el cambio de color) o la absorbancia neta para así relacionarlo con la dosis de irradiación.

Un sistema óptico consiste, básicamente, en una fuente de luz y un detector capaz de percibir esta luz transmitida por un medio, en este caso en particular el medio es la película radiocrómica. Por lo tanto, con un sistema óptico se puede medir la intensidad de luz transmitida (I) a través de la película radiocrómica y calcular la absorbancia neta mediante la siguiente Ecuación:

$$
A_{\text {neta }}(\lambda)=-\log _{10}\left(\frac{I_{i}(\lambda)}{I_{0}(\lambda)}\right)
$$

donde $I_{i}$ y $I_{o}$ son las intensidades transmitidas por las películas radiocrómicas irradiada y no irradiada (o $G y$ ) respectivamente, como una función de la longitud de onda $(\lambda)$ de la fuente de luz que utiliza el sistema óptico.

Existen varios sistemas ópticos que se pueden utilizar para medir la respuesta de las películas radiocrómicas, sin embargo, los más utilizados son los escáneres de cama plana con fuente de luz fluorescente o diodo emisor de luz (LED por sus siglas en inglés). Además, se pueden utilizar los espectrofotómetros operando a $636 \mathrm{~nm}$ para obtener los más altos niveles de sensibilidad en la respuesta, debido a que trabajan en el pico de absorción de las películas EBT-EBT3. También se pueden leer con un LED de haz estrecho con anchura total a la mitad del máximo (FWHM por sus siglas en inglés) alrededor de $20 \mathrm{~nm}$ o utilizando un láser de Helio-Neón (He-Ne) con banda de emisión en $632.8 \mathrm{~nm}$ (rojo). Cada uno de estos sistemas tiene asociada una sensibilidad que depende de la longitud de onda o banda ancha que utilice la fuente de luz ${ }^{[4]}$, la cual influye en la respuesta de la película radiocrómica. Debido a esto, el estudio del espectro de absorción de las películas radiocrómicas es un factor importante dentro de la caracterización de ésta como dosímetro de referencia, ya que favorece la elección del mejor sistema óptico para su análisis, por otro lado, puede ayudar a su vez, a desarrollar nuevos sistemas ópticos mejorados para ampliar el uso de las películas radiocrómicas ${ }^{[5]}$.

Por ello, este trabajo pretende difundir como área de oportunidad, el estudio y análisis de los espectros de 
absorción de las películas radiocrómicas como una fuente de generación de nuevos conocimientos para una posible creación de nuevos sistemas ópticos capaces de amplificar la sensibilidad de la respuesta de las películas con el simple hecho de estudiar y analizar el comportamiento de los espectros de absorción de estos modelos de películas. Por ejemplo, al saber dónde se encuentran los picos de absorción en función de la dosis, se pueden generar sistemas ópticos capaces de trabajar en esa longitud de onda determinada, amplificando con ello la sensibilidad en la respuesta de las películas que el sistema óptico es capaz de interpretar, favoreciendo el uso clínico de este modelo de detectores de radiación. Lo que conlleva a un rango dinámico de uso clínico mayor, no solamente en aplicaciones, si no inclusive en las mismas aplicaciones ya conocidas, pero con un intervalo útil de dosis mayor.

El objetivo de este trabajo es presentar, analizar y comparar los espectros de absorción neta de las películas radiocrómicas EBT2 у EBT3 irradiadas de 0 a $10 \mathrm{~Gy}$, utilizando un espectrofotómetro UV/VIS de doble haz. Además, se describirá la influencia del comportamiento del espectro de absorción de las películas radiocrómicas EBT2 y EBT3 en la construcción de las curvas de dosis-respuesta, utilizando tres sistemas ópticos con diferente longitud de onda o ancho de banda: un escáner, un láser He-Ne y un espectrofotómetro. Este análisis en las curvas de dosis-respuesta en función del espectro, repercuten directamente en la clínica, ya que los rangos dinámicos en dosis están directamente relacionados con su sensibilidad y por tanto al uso del sistema óptico empleado.

\section{METODOLOGÍA}

\section{Irradiación de las películas radiocrómicas}

Para el desarrollo de este trabajo se utilizaron piezas de película radiocrómica EBT2 (Lot\# A12171003B) y EBT3 (Lot\# A01171301) con un tamaño de $3 \mathrm{~cm} \times 3 \mathrm{~cm}$. Las películas radiocrómicas se irradiaron con un acel- erador lineal (LINAC) Novalis ${ }^{\circledR}$ BrainLab de $6 \mathrm{MV}$ en modo de fotones, colocándolas a $5 \mathrm{~cm}$ de profundidad dentro de un maniquí de agua sólida Plastic Water en forma perpendicular al haz de radiación. El LINAC fue calibrado para impartir 1 cGy por unidad monitor, utilizando un campo de $10 \mathrm{~cm} \times 10 \mathrm{~cm}$ y una distancia fuente superficie (SSD por sus siglas en inglés) de 95 $\mathrm{cm}$. Las películas radiocrómicas EBT2 y EBT3 fueron irradiadas en un intervalo de dosis de 0 a $10 \mathrm{~Gy}$, considerando las siguientes dosis: $0,0.25,0.5,1,1.5,2,3,5$, 7 y $10 \mathrm{~Gy}$. Con la finalidad de reducir la incertidumbre se consideraron 3 películas radiocrómicas para cada dosis, trabajando finalmente, con el promedio de la respuesta de las tres películas radiocrómicas ${ }^{[6]}$.

\section{Espectros de absorción neta de las películas radiocrómicas}

Los espectros de absorción neta de las películas radiocrómicas EBT2 y EBT3 se obtuvieron utilizando un espectrofotómetro UV/VIS de doble haz marca Perkin Elmer modelo Lambda 650. Los espectros se midieron para un intervalo de longitudes de onda de $400 \mathrm{~nm}$ a 800 $\mathrm{nm}$ con pasos de $1 \mathrm{~nm}$ y una precisión de $\pm 0.15 \mathrm{~nm}{ }^{[7]}$. El equipo se encendió 30 minutos antes de iniciar la lectura con la finalidad de que la fuente de luz se estabilizara. Debido a que el espectrofotómetro es un equipo de doble haz, proporciona los valores de la absorbancia neta. Por lo tanto, se debe colocar la película radiocrómica sin irradiar (o Gy) en el porta muestras de referencia y, en el otro porta muestras, se colocan las películas radiocrómicas irradiadas entre 0 y $10 \mathrm{~Gy}$ (una por una).

\section{Curvas de dosis-respuesta de las películas radiocrómicas}

Una curva de dosis-respuesta es una representación gráfica de la respuesta de la película radiocrómica $\left(A_{\text {neta }} \mathrm{o}\right.$ $D O_{n e t a}$ ) en función de la dosis. Para determinar la absorbancia neta utilizando la Ecuación anteriormente definida es necesario medir la intensidad transmitida a través de la película radiocrómica irradiada $\left(I_{i}\right)$ y no irradiada $\left(I_{0}\right)$ con los dos sistemas ópticos: escáner y láser de He-Ne. Por su 
parte, el espectrofotómetro proporciona valores de absorbancia neta (como se describió en el apartado de espectros de absorción neta de las películas radiocrómicas).

\section{Escáner: lectura y procesamiento de imágenes}

En este trabajo se utilizó un escáner de cama plana Epson Perfection V750 con su software asociado Epson Scan (Epson, Nagano, Japón). El escáner utiliza una fuente de luz blanca. Las películas radiocrómicas se escanearon en modo de transmisión, en formato rojo, verde y azul (RGB por sus siglas en inglés) con 48 bit de profundidad de color y todas las opciones de procesamiento de imágenes desactivadas. Se utilizó una resolución de 72 puntos por pulgada ( $p p p)$. Todas las películas radiocrómicas se escanearon en la posición central de la cama del escáner y en orientación portrait ${ }^{[8]}$. Alrededor de la película se colocó una máscara negra con la finalidad de evitar la contribución de la luz dispersa por la lámpara del escáner a la respuesta de la película. Antes de iniciar los escaneos, se estabilizó la lámpara del escáner por 15 minutos. Las imágenes se guardaron en formato TIFF para su procesamiento, el cual se realizó con el software ImageJ (v.1.2). El análisis solo se efectuó para la componente roja [9], obteniendo la intensidad transmitida tanto de las películas radiocrómicas irradiadas $\left(I_{i}\right)$ como de la no irradiada $\left(I_{0}\right)$ y la desviación estándar asociada a estas, $D E\left(I_{i}\right)$ y $D E\left(I_{0}\right)$, respectivamente.

\section{Láser: medición de la intensidad transmitida}

Para medir la intensidad transmitida a través de las películas radiocrómicas irradiadas y la no irradiada utilizamos un láser He-Ne de emisión continua a una longitud de onda de $633 \mathrm{~nm}$. Esta longitud de onda se encuentra en el intervalo del rojo $(622 \mathrm{~nm}-780 \mathrm{~nm})$ del espectro visible $(390 \mathrm{~nm}-780 \mathrm{~nm})^{[10]}$. La luz emitida por el láser incide en la película radiocrómica y la luz trasmitida a través de la película llega a un detector, fotodiodo de silicio EOT modelo ET-2040. La inten- sidad trasmitida $\left(I_{i}\right.$ e $\left.I_{o}\right)$ se registra en un voltímetro que se encuentra conectado al detector.

\section{Espectrofotómetro: obtención de la absorbancia neta}

Del espectro de absorción neta de las películas radiocrómicas se extrae la absorbancia neta para cada dosis a una longitud de onda de $636 \mathrm{~nm}$, donde se encuentra el pico de absorción de las películas radiocrómicas EBT2 [11].

\section{RESULTADOS}

Las Figuras 1 y 2 muestran los espectros de absorción neta de las películas radiocrómicas EBT2 y EBT3 respectivamente, para un intervalo de longitudes de onda de 400 a $800 \mathrm{~nm}$ e irradiadas en un intervalo de dosis de 0 a $10 \mathrm{~Gy}$. Como puede observarse, los espectros de absorción neta de las películas radiocrómicas EBT2 presenta una banda de absorción principal centrada alrededor de $636 \mathrm{~nm}$ y dos bandas de absorción secundarias centradas alrededor de 583 y $565 \mathrm{~nm}$. La posición de estas bandas se mantiene para todos los niveles de dosis estudiados. Además, la absorbancia neta aumenta como una función de la dosis. Por otro lado, en los espectros de absorción neta de las películas radiocrómicas EBT3, también se observan estas tres bandas de absorción. Sin embargo, estas bandas de absorción no mantienen la posición del máximo al variar la dosis. El máximo de la banda de absorción principal se encuentra centrado alrededor de un intervalo de longitudes de onda entre 635 y $639 \mathrm{~nm}$, mientras que el máximo de las bandas de absorción secundarias se localiza alrededor del intervalo de longitudes de onda de 581-588 $\mathrm{nm}$ y 561-566 $\mathrm{nm}$, respectivamente. Además, en los espectros de absorción neta de las películas radiocrómicas EBT2 se observan tres bandas de absorción más, a longitudes de onda más cortas, centradas alrededor de: 509, 455 y $421 \mathrm{~nm}$. Mientras que, en los espectros de absorción neta de las películas radiocrómicas EBT3 se observa una serie de bandas en el intervalo de longitudes de onda de 400 a $550 \mathrm{~nm}$. 


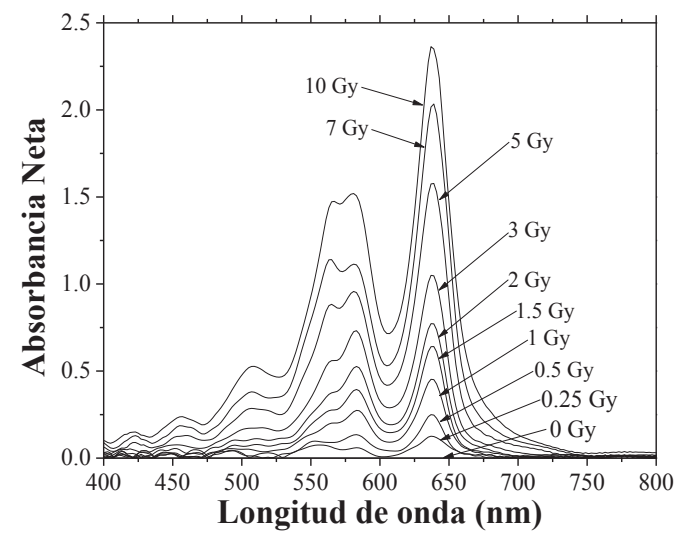

FIGURA 1. Espectros de absorción neta de las PR EBT2 irradiadas en un intervalo de dosis de 0 a $10 \mathrm{~Gy}$.

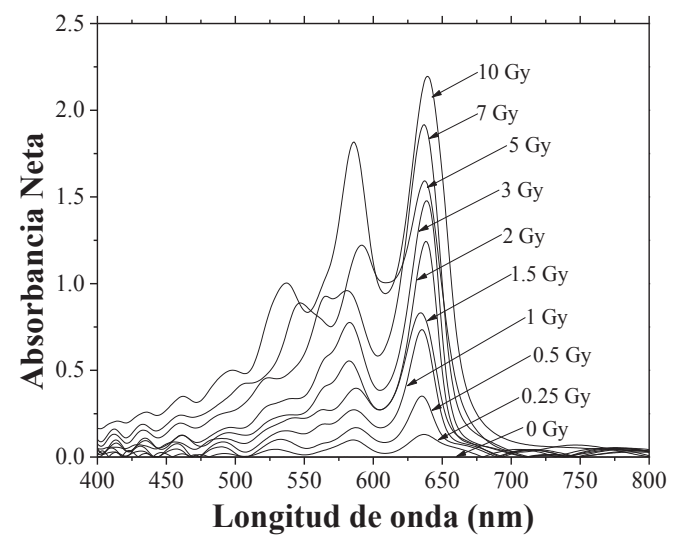

FIGURA 2. Espectros de absorción neta de la PR EBT3 irradiadas en un intervalo de dosis de 0 a $10 \mathrm{~Gy}$.

En las Figuras 3 y 4 se presentan las curvas de dosis-respuesta de las películas radiocrómicas EBT2 y EBR3 irradiadas en un intervalo de dosis de 0 a 10 Gy y analizada con tres diferentes sistemas ópticos: escáner, láser y espectrofotómetro. Como puede observarse, la máxima sensibilidad en la respuesta de las películas radiocrómicas EBT2 y EBT3 se obtiene al utilizar un espectrofotómetro como sistema de lectura. Esto se debe a que, al centrarse en la banda de absorbancia neta se toma el valor máximo que puede tener la absorbancia para cada dosis. A este le sigue el láser, cuya sensibilidad es menor debido a que se considera la absorbancia a $633 \mathrm{~nm}$, es decir, a $3 \mathrm{~nm}$ del centro de la banda de absorbancia, pero sigue teniendo buena sensibilidad. Por otro lado, observamos que al leer las películas radiocrómicas con un escáner tenemos la mínima sensibilidad en la respuesta, respecto a los otros dos sistemas ópticos.

La razón de esta disminución en la sensibilidad de la respuesta de las películas radiocrómicas es debido a que el escáner utiliza una fuente de luz blanca y al analizar la absorbancia en el canal rojo se considera un ancho de banda de $100 \mathrm{~nm}(600 \text { a } 700 \mathrm{~nm})^{[12]}$. Sin embargo, es de notarse que las curvas de dosis-respuesta de la película radiocrómica EBT3 obtenidas con el láser y el espectrofotómetro no presentan un comportamiento tan bien definido como las curvas de dosis-respuesta de las películas radiocrómicas EBT2, el cual si se observa al utilizar el escáner.

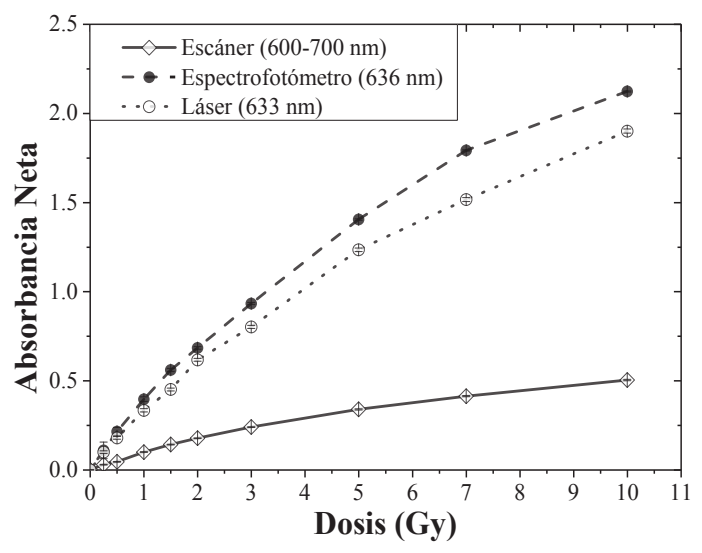

FIGURA 3. Curvas de dosis-respuesta de la PR EBT2 irradiadas en un intervalo de dosis de 0 a $10 \mathrm{~Gy}$ y analizada con diferentes sistemas ópticos. 


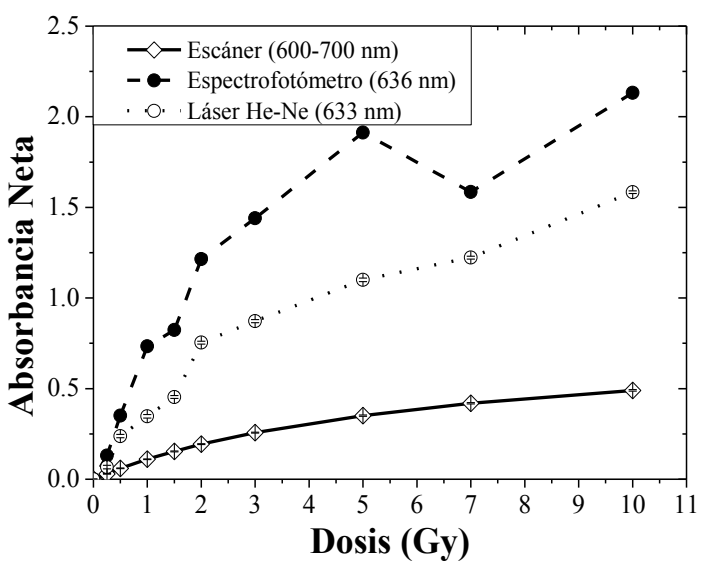

FIGURA 4. Curvas de dosis-respuesta de la PR EBT3 irradiadas en un intervalo de dosis de 0 a $10 \mathrm{~Gy}$ y analizada con diferentes sistemas ópticos.

\section{DISCUSIÓN}

Las bandas de absorción que presentan los espectros de las películas radiocrómicas EBT2 se muestran bien definidas para cada dosis estudiada, aumentando la absorbancia neta como una función de la dosis. Este comportamiento de los espectros de absorción neta de las películas radiocrómicas EBT2 es consistente con lo reportado para los espectros de absorción de las películas radiocrómicas EBT2 ${ }^{[12]}$ y EBT ${ }^{[5,11,13]}$. Por otra parte, se esperaría que los espectros de absorción neta de las películas radiocrómicas EBT3 no modificara su comportamiento respecto los espectros de absorción neta de las películas radiocrómicas EBT2, debido a que la composición química de la capa activa de las películas radiocrómicas EBT3 es similar que la de su predecesora, las películas radiocrómicas EBT2. Sin embargo, existen dos diferencias notables, una es el desplazamiento de las bandas de absorción y la otra es el comportamiento del espectro de absorción neta para el intervalo de longitudes de onda de 400 a $550 \mathrm{~nm}$ aproximadamente. Este comportamiento de los espectros de absorción de las películas radiocrómicas se le puede atribuir a un fenómeno de interferencia característico de una cavidad de Fabry-Perot formado por la estructura simétrica de las películas radiocrómicas EBT3 donde las capas de poliéster representan los espejos semireflectores y la capa activa funge como la cavidad resonante ${ }^{[7]}$.
Como se mencionó anteriormente el resultado de los espectros de absorción de las películas radiocrómicas EBT2 y EBT3 influye directamente en la elección del sistema óptico que se utilizará para el análisis de la respuesta (absorbancia neta o densidad óptica neta). Por lo cual, analizando las curvas de dosis-respuesta; si deseamos tener la máxima sensibilidad, elegimos un espectrofotómetro operando a la longitud de onda del máximo de la banda de absorción o un láser de He-Ne. Esto de acuerdo con la Ecuación anteriormente definida, la absorbancia neta depende de la longitud de onda que tiene la luz que utiliza el sistema óptico. Para el caso del espectrofotómetro, la absorbancia se toma a $636 \mathrm{~nm}$ (pico de absorción), mientras que el láser emite luz a $633 \mathrm{~nm}$. Sin embargo, para las películas radiocrómicas EBT3, las curvas de dosis-respuesta de estos sistemas ópticos no muestran un comportamiento definido. Por lo tanto, se dificultaría la construcción de una curva de respuesta y esto aumentaría la incertidumbre en el proceso de calibración y su uso en QA en radioterapia. Este comportamiento se puede deber al desplazamiento que presentan las bandas de absorción de las películas radiocrómicas EBT3, ya que el máximo valor de la absorbancia no siempre se obtiene a los $636 \mathrm{~nm}$. Por el contrario, debido al comportamiento de los espectros de absorción neta de las películas radiocrómicas EBT2, es posible utilizar estos sistemas ópticos para el análisis de la respuesta película y su uso en QA en radioterapia.

Para el caso del escáner, el cual utiliza una fuente de luz blanca, con un ancho de banda de $100 \mathrm{~nm}$, aproximadamente, la absorbancia neta es el resultado de la convolución del espectro de emisión de la fuente de luz ${ }^{[5]}$. Por esta razón, cuando las películas radiocrómicas se analizan con un escáner, la sensibilidad de la respuesta disminuye considerablemente con respecto a la sensibilidad que presenta la respuesta de las películas radiocrómicas al analizarse con un espectrofotómetro o un láser. Pero a diferencia de estos sistemas ópticos, las curvas de dosis-respuesta obtenidas 
con el escáner presentan un comportamiento bien definido, tanto para las películas radiocrómicas EBT2 como para las películas radiocrómicas EBT3. Esto facilita que a las curvas de dosis-respuesta se les asocie una función de ajuste que describa a la dosis en función de la respuesta (absorbancia neta o densidad óptica neta) dando como resultado una desviación razonable para su uso en QA en radioterapia.

\section{CONCLUSIÓN}

Los espectros de absorción neta de las películas radiocrómicas EBT2 presenta tres bandas de absorción intensas centradas alrededor de $636 \mathrm{~nm}$, la más intensa y las de menor intensidad centradas alrededor de 583 y $565 \mathrm{~nm}$ aproximadamente. La posición de estas bandas se mantiene para un intervalo de dosis de 0 a $10 \mathrm{~Gy}$ aunado a que la absorbancia neta aumenta como una función de la dosis. Para los espectros de absorción neta de las películas radiocrómicas EBT3, también se observan estas tres bandas de absorción, pero, a diferencias de las películas radiocrómicas EBT2, no conservan su posición. Esto se puede atribuir a un efecto de interferencia de Fabry-Perot, producto de la estructura simétrica de la película radiocrómica EBT3. Este comportamiento de los espectros de las películas radiocrómicas EBT3 influye en la elección del sistema óptico con el que se analiza la respuesta de la película radiocrómica. Dando lugar a la elección del escáner de cama plana para el uso de QA en radioterapia, ya que a pesar de poseer en menor rango de sensibilidad muestra el mejor comportamiento en la curva dosis-respuesta por lo señalado en la sección de discusión.

Reiterando con ello, la importancia de realizar un análisis de los espectros de absorción de las películas radiocrómicas como lo propone este trabajo, para con ello, primero entender el comportamiento del mismo y segundo descartar el uso de sistemas ópticos que no garanticen un uso clínico confiable. Aunado a que, estos resultados dan pauta para el desarrollo de nuevos sistemas ópticos capaces de mantener un rango sensibilidad óptimo, pero a su vez mantener el comportamiento de la curva dosis-respuesta deseable para ser utilizado en control de calidad y aplicaciones médicas. 


\section{REFERENCIAS}

[1] Casanova-Borca V, Pasquino M, Russo G, Grosso P, Cante D, Sciacero P, et al., "Dosimetric characterization and use of GAFCHROMIC EBT3 film for IMRT dose verification,” J Appl Clin Med Phys., vol. 14, no. 2, pp. 158-171, 2013. http://dx.doi. org/10.1120/jacmp.v14i2.4111

[2] Lewis D, Micke A, Yu X, Chan MF, "An efficient protocol for radiochromic film dosimetry combining calibration and measurement in a single scan,” Med Phys., vol. 39, no. 10, pp. 6339-6350, 2012. DOI: http://dx.doi.org/10.1118/1.4754797.

[3] Aland T, Kairn T, Kenny J, "Evaluation of a Gafchromic EBT2 film dosimetry system for radiotherapy quality assurance," Australas Phys Eng Sci Med., vol. 34, pp. 251-260, 2011. DOI: 10.1007/ s13246-011-0072-6

[4] Devic S, Seuntjens J, Sham E, Podgorsak EB, Schmidtlein CR, Kirov AS, et al., "Precise radiochromic film dosimetry using a flat-bed document scanner,” Med. Phys., vol. 32, pp. 2245-2253, 2005. DOI: $10.1118 / 1.1929253$

[5] Devic S, Tomic N, Pang Z, Seuntjens J, Podgorsak EB, Soares CG, "Absorption spectroscopy of EBT model GAFCHROMICTM film," Med. Phys., vol. 34, pp. 112-118, 2007. DOI:10.1118/1.2400615

[6] Devic S, Wang YZ, Tomic N, Podgorsak EB, "Sensitivity of linear CCD array based film scanners used for film dosimetry," Med Phys., vol. 33, no. 11, pp. 3993-3996, 2006. DOI: http://dx.doi. org/10.1118/1.2357836
[7] León-Marroquín EY, Camacho-López MA, García-Garduño OA, Herrera-González JA, Villarreal-Barajas JE, Gutiérrez-Fuentes R, et al., "Spectral analysis of the EBT3 radiochromic film irradiated with 6 MV x-ray radiation,” Radiat. Meas., vol. 89, pp. 82-88, 2016. DOI: http://dx.doi.org/10.1016/j.radmeas.2016.03.007

[8] León-Marroquín EY; Herrera-González JA; Camacho-López MA; Villarreal-Barajas JE; García-Garduño OA, "Evaluation of the uncertainty in a EBT3 film dosimetry system utilizing net optical density,” J. Appl. Clin. Med. Phys., vol. 17, no. 5, pp. 466-481, 2016. DOl: http://dx.doi.org/10.1120/jacmp.v17i5.6262

[9] Devic S, "Radiochromic film dosimetry: Past, present and future," Phys. Med., vol. 27, pp. 122-134, 2011. DOI: http://dx.doi. org/10.1016/i.ejmp.2010.10.001

[10] Hecht. Óptica, Pearson (España), pp. 77-78, 2000.

[11] Butson MJ, Cheung T, Yu PKN, Alnawaf H, "Dose and absorption spectra response of EBT2 Gafchromic film to high energy x-rays," Australas. Phys. Eng. Sci. Med., vol. 32, pp. 196-202, 2009. DOI:10.1007/BF03179239

[12] Mack A, Mack G, Weltz D, Sxheib SG, Böttcher HD, Seifert V, "High precision film dosimetry with GafChromic films for quality assurance especially when using small fields," Med. Phys., vol. 30, no. 9, pp. 2399-2409, 2003. DOI: 10.1118/1.1593634

[13] Butson MJ, Cheung T, Yu PKN, "Absorption spectra variations of EBT radiochromic film from radiation exposure," Phys. Med. Biol., vol. 50, pp. N135-N140, 2005. DOl: http://dx.doi.org/10.1088/0031$\underline{9155 / 50 / 13 / \mathrm{NO}}$ 